\title{
APPLICATIONS OF AN INEQUALITY FOR THE SCHUR COMPLEMENT
}

EMILIE V. HAYNSWORTH

1. Introduction. Suppose $B$ is a nonsingular principal submatrix of an $n \times n$ matrix $A$. The Schur Complement of $B$ in $A$, denoted by $(A / B)$, is defined as follows: Let $\hat{A}$ be the matrix obtained from $A$ by the simultaneous permutation of rows and columns which puts $B$ into the upper left corner of $\hat{A}$,

$$
A=\left(\begin{array}{ll}
B & C \\
D & G
\end{array}\right)
$$

leaving the rows and columns of $B$ and $G$ in the same increasing order as in $A$. Then the Schur Complement of $B$ in $A$ is

$$
(A / B)=G-D B^{-1} C .
$$

Schur proved that the determinant of $A$ is the product of the determinant of any nonsingular principal submatrix $B$ with that of its Schur complement,

$$
|A|=|B||(A / B)| \text {. }
$$

The inertia of an Hermitian matrix $A$ is given by the ordered triplet, In $A=(\pi, \nu, \delta)$, where $\pi$ denotes the number of positive, $\nu$ the number of negative, and $\delta$ the number of zero roots of the matrix $A$. In a previous paper [2], it was shown that the inertia of an Hermitian matrix can be determined from that of any nonsingular principal submatrix together with that of its Schur complement. That is, if $A$ is Hermitian, and $B$ is a nonsingular principal submatrix of $A$, then

$$
\text { In } A=\operatorname{In} B+\operatorname{In}(A / B) \text {. }
$$

More recently, the author, with Douglas Crabtree [1], proved the identity,

$$
(A / B)=((A / C) /(B / C)) .
$$

In Theorem 1 of $\$ 2$ we make use of (3) to prove an extension of a theorem by Marcus [3]. Then in Theorem 2 we apply the result of Theorem 1 to obtain an inequality for the Schur complement which is similar to Minkowski's famous inequality (see [4]) for the determinant of the sum of positive definite Hermitian matrices:

Received by the editors May 16, 1969. 


$$
|A+B|^{1 / n} \geqq|A|^{1 / n}+|B|^{1 / n} \quad \text { (Minkowski). }
$$

This, of course, implies

$$
|A+B| \geqq|A|+|B| \text {. }
$$

A number of extensions of the Minkowski inequality have been proved by Marcus, Minc and others (see [5]).

In Theorem 3 we obtain some new inequalities for the determinant of the sum of two positive definite Hermitian matrices.

2. An extension of a theorem by Marcus. In a recent paper [3] $M$. Marcus proved a number of interesting inequalities for positive definite Hermitian matrices, including the following: If $H$ and $K$ are positive definite matrices of order $n$, and $X$ and $Y$ are arbitrary vectors, then

$$
\left(H^{-1} X, X\right)+\left(K^{-1} Y, Y\right) \geqq\left((H+K)^{-1}(X+Y),(X+Y)\right) .
$$

It is shown in Theorem 1 that by making use of the properties of the Schur complement this inequality can be extended to the case where $X$ and $Y$ are arbitrary $n \times m$ matrices. We shall use the notation $A \geqq 0$ for a positive semidefinite matrix (p.s.d. matrix), with strict inequality implying that $A$ is positive definite (p.d.). If $A$ and $B$ are p.s.d. matrices, the statement $A \geqq B$ will mean $A-B \geqq 0$.

Theorem 1. Suppose $H$ and $K$ are positive definite matrices of order $n$. Then if $X$ and $Y$ are arbitrary $n \times m$ matrices, the $m \times m$ matrix

$$
Q=X^{*} H^{-1} X+Y^{*} K^{-1} Y-(X+Y)^{*}(H+K)^{-1}(X+Y)
$$

is positive semidefinite.

Proof. Let $A$ and $B$ be the Hermitian matrices of order $2 n$,

$$
A=\left(\begin{array}{cc}
H & X \\
X^{*} & X^{*} H^{-1} X
\end{array}\right), \quad B=\left(\begin{array}{cc}
K & Y \\
Y^{*} & Y^{*} K^{-1} Y
\end{array}\right) .
$$

From (3), it is clear that a nonzero Hermitian matrix is positive semidefinite (definite) if and only if there exists a positive definite principal submatrix whose Schur complement is positive semidefinite (definite). Thus, by inspection, the matrices $A$ and $B$ are positive semidefinite. Then, since the sum of any two positive semidefinite matrices is also positive semidefinite (or definite) we have

$$
A+B=\left(\begin{array}{lc}
H+K & X+Y \\
X^{*}+Y^{*} & X^{*} H^{-1} X+Y^{*} K^{-1} Y
\end{array}\right) \geqq 0 .
$$


This proves the theorem, as the matrix $Q$ in (5) is the Schur complement of $H+K$ in $A+B$.

\section{An inequality for the Schur complement.}

Tineorem 2. Suppose $A$ and $B$ are Hermitian matrices of order $n$, partitioned into $2 \times 2$ block matrices, $A=\left(A_{i j}\right), B=\left(B_{i j}\right), i, j=1,2$, where $A_{11}$ and $B_{11}$ are square of order $m$. If $A \geqq 0, B \geqq 0, A_{11}>0, B_{11}>0$, then

$$
\left(A+B / A_{11}+B_{11}\right) \geqq\left(A / A_{11}\right)+\left(B / B_{11}\right) .
$$

Proof. By the previous arguments, $A_{11}+B_{11}>0$, and $A+B \geqq 0$. From the definition,

$$
\begin{aligned}
\left(A+B / A_{11}+B_{11}\right)= & \left(A_{22}+B_{22}\right)-\left(A_{21}+B_{21}\right)\left(A_{11}+B_{11}\right)^{-1} \\
& \cdot\left(A_{12}+B_{12}\right) .
\end{aligned}
$$

By Theorem 1,

$$
\left(A_{21}+B_{21}\right)\left(A_{11}+B_{11}\right)^{-1}\left(A_{12}+B_{12}\right) \leqq A_{21} A_{11}^{-1} A_{12}+B_{21} B_{11}^{-1} B_{12} .
$$

Thus

$$
\begin{aligned}
\left(A+B / A_{11}+B_{11}\right) & \geqq\left(A_{22}+B_{22}\right)-\left(A_{21} A_{11}^{-1} A_{12}+B_{21} B_{11}^{-1} B_{12}\right) \\
& =\left(A / A_{11}\right)+\left(B / B_{11}\right) .
\end{aligned}
$$

This proves the formula (6), which we now apply to find a new inequality for the determinant of the sum of two positive definite Hermitian matrices.

\section{Some determinantal inequalities.}

Theorem 3. Suppose $A$ and $B$ are positive definite Hermitian matrices. Let $A_{k}$ and $B_{k}, k=1, \cdots, n$, denote the principal submatrices of order $k$ in the upper left corner of the matrices $A$ and $B$ respectively. Then

$$
|A+B| \geqq|A|\left(1+\sum_{k=1}^{n-1} \frac{\left|B_{k}\right|}{\left|A_{k}\right|}\right)+|B|\left(1+\sum_{k=1}^{n-1} \frac{\left|A_{k}\right|}{\left|B_{k}\right|}\right) \text {. }
$$

Corollary. If $A$ and $B$ are positive definite, and $A>B$, then

$$
|A+B|>|A|+n|B| \text {. }
$$

For the proof of Theorem 3 we need the following lemmas. Lemma 1 is probably well known, as it follows immediately from the Minkowski inequality (4). Lemma 2 follows as a corollary to Lemma 1 and Theorem 2. 
Lemma 1. If $A$ and $B$ are positive definite Hermitian matrices and $A>B$, then $\left|A_{k}\right|>\left|B_{k}\right|, k=1, \cdots, n$.

Proof. Let $A-B=C>0$. Then $A_{k}=B_{k}+C_{k}(k=1, \cdots, n)$ where $A_{k}, B_{k}$, and $C_{k}$ are positive definite, since they are principal submatrices of positive definite matrices. Then by (4), $\left|A_{k}\right| \geqq\left|B_{k}\right|+\left|C_{k}\right|$ $>\left|B_{k}\right|(k=1, \cdots, n)$.

Lemma 2. If $A$ and $B$ satisfy the conditions of Theorem 2, then

$$
\left|\left(A+B / A_{11}+B_{11}\right)\right| \geqq|A| /\left|A_{11}\right|+|B| /\left|B_{11}\right| \text {. }
$$

Proof. By Theorem 2 and Lemma 1,

$$
\begin{array}{rlrl}
\left|\left(A+B / A_{11}+B_{11}\right)\right| & \geqq\left|\left(A / A_{11}\right)+\left(B / B_{11}\right)\right| & \\
& \geqq\left|\left(A / A_{11}\right)\right|+\left|\left(B / B_{11}\right)\right| & & \text { by (4) } \\
& =|A| /\left|A_{11}\right|+|B| /\left|B_{11}\right| & & \text { by (2). }
\end{array}
$$

Proof of Theorem 3. We prove the theorem by induction on $n$. For $n=2$, we have from (2),

$$
|A+B|=\left|A_{1}+B_{1}\right|\left|\left(A+B / A_{1}+B_{1}\right)\right| \text {. }
$$

By Lemma 2,

$$
\left|\left(A+B / A_{1}+B_{1}\right)\right| \geqq|A| /\left|A_{1}\right|+|B| /\left|B_{1}\right| .
$$

Thus, using (4) on the first factor on the right in (9),

$$
|A+B| \geqq\left(\left|A_{1}\right|+\left|B_{1}\right|\right)\left(|A| /\left|A_{1}\right|+|B| /\left|B_{1}\right|\right)
$$

which proves (7) for $n=2$.

Now assume (7) holds for matrices of order less than or equal to $n-1$. Then, if $A$ and $B$ are of order $n$,

$$
|A+B| \geqq\left(\left|A_{n-1}+B_{n-1}\right|\right)\left|\left(A+B / A_{n-1}+B_{n-1}\right)\right|,
$$

where, by the inductive assumption,

$$
\begin{aligned}
& \left|A_{n-1}+B_{n-1}\right| \\
& \quad \geqq\left|A_{n-1}\right|\left(1+\sum_{k=1}^{n-2} \frac{\left|B_{k}\right|}{\left|A_{k}\right|}\right)+\left|B_{n-1}\right|\left(1+\sum_{k=1}^{n-2} \frac{\left|A_{k}\right|}{\left|B_{k}\right|}\right),
\end{aligned}
$$

and, by Lemma 2 ,

$$
\left|\left(A+B / A_{n-1}+B_{n-1}\right)\right| \geqq|A| /\left|A_{n-1}\right|+|B| /\left|B_{n-1}\right| .
$$

Thus 


$$
\begin{aligned}
|A+B| \geqq( & \left|A_{n-1}\right|\left(1+\sum_{k=1}^{n-2} \frac{\left|B_{k}\right|}{\left|A_{k}\right|}\right) \\
& \left.+\left|B_{n-1}\right|\left(1+\sum_{k=1}^{n-2} \frac{\left|A_{k}\right|}{\left|B_{k}\right|}\right)\right)\left(\frac{|A|}{\left|A_{n-1}\right|}+\frac{|B|}{\left|B_{n-1}\right|}\right) \\
=|A| & \left(1+\sum_{k=1}^{n-2} \frac{\left|B_{k}\right|}{\left|A_{k}\right|}\right)+|B|\left(1+\sum_{k=1}^{n-2} \frac{\left|A_{k}\right|}{\left|B_{k}\right|}\right) \\
& +\left|\frac{A_{n-1}}{B_{n-1} \mid}\right|\left(1+\sum_{k=1}^{n-2} \frac{\left|B_{k}\right|}{\left|A_{k}\right|}\right)|B| \\
& +\left|\frac{B_{n-1}}{A_{n-1}}\right|\left(1+\sum_{k=1}^{n-2} \frac{\left|A_{k}\right|}{\left|B_{k}\right|}\right)|A| \\
\geqq \mid & \left|\left(1+\sum_{k=1}^{n-1} \frac{\left|B_{k}\right|}{\left|A_{k}\right|}\right)+\right| B \mid\left(1+\sum_{k=1}^{n-1} \frac{\left|A_{k}\right|}{\left|B_{k}\right|}\right) .
\end{aligned}
$$

This proves Theorem 3 .

The corollary follows as an immediate consequence of Lemma 1, since if $A>B$,

$$
\left|A_{k}\right| /\left|B_{k}\right|>1 \quad(k=1, \cdots, n) .
$$

Hence

$$
|A+B| \geqq|A|\left(1+\sum_{k=1}^{n-1} \frac{\left|B_{k}\right|}{\left|A_{k}\right|}\right)+n|B| \geqq|A|+n|B| .
$$

Acknowledgments. This work was done under contract DA-91591-EUC-3686 of the U.S. Army with the Institute of Mathematics, University of Basel. The author wishes to thank Professor A. M. Ostrowski for very helpful discussions.

\section{REFERENCES}

1. D. Crabtree and E. Haynsworth, An identity for the Schur complement of $a$ matrix, Proc. Amer. Math. Soc. 22(1969), 364-366.

2. E. Haynsworth, Determination of the inertia of a partitioned Hermitian matrix, Linear Algebra and Appl. 1 (1968), no. 1, 73-81. MR 36 \#6440.

3. M. Marcus, An inequality for linear transformations, Proc. Amer. Math. Soc. 18 (1967) 793-797. MR 36 \#3811.

4. M. Marcus and H. Minc, A survey of matrix theory and matrix inequalities, Allyn \& Bacon, Boston, Mass., 1964, p. 115. MR 29 \#112.

5. M. Marcus and S. Pierce, Extensions of the Minkowski inequality, Linear Algebra and Appl. 1 (1968), no. 1, 13-27. MR 36 \#6431.

Auburn University 